\title{
Dexmedetomidine is neuroprotective in an in vitro model for traumatic brain injury
}

\author{
Marc Schoeler ${ }^{1}$, Philip D Loetscher ${ }^{1}$, Rolf Rossaint ${ }^{1}$, Astrid V Fahlenkamp ${ }^{1}$, Georg Eberhardt ${ }^{1}$, Steffen Rex ${ }^{1,3}$, \\ Joachim Weis $^{2}$ and Mark Coburn ${ }^{1 *}$
}

\begin{abstract}
Background: The $\alpha_{2}$-adrenoreceptor agonist dexmedetomidine is known to provide neuroprotection under ischemic conditions. In this study we investigated whether dexmedetomidine has a protective effect in an in vitro model for traumatic brain injury.

Methods: Organotypic hippocampal slice cultures were subjected to a focal mechanical trauma and then exposed to varying concentrations of dexmedetomidine. After $72 \mathrm{~h}$ cell injury was assessed using propidium iodide. In addition, the effects of delayed dexmedetomidine application, of hypothermia and canonical signalling pathway inhibitors were examined.
\end{abstract}

Results: Dexmedetomidine showed a protective effect on traumatically injured hippocampal cells with a maximum effect at a dosage of $1 \mu \mathrm{M}$. This effect was partially reversed by the simultaneous administration of the ERK inhibitor PD98059.

Conclusion: In this TBI model dexmedetomidine had a significant neuroprotective effect. Our results indicate that activation of ERK might be involved in mediating this effect.

Keywords: Neuroprotection, Traumatic brain injury, Dexmedetomidine

\section{Background}

In the United States an estimated 1.1 million patients are treated for traumatic brain injury (TBI) annually [1]. Of these, 124.000 patients suffer from long-term disabilities and 50.000 die, which makes TBI a major cause of premature death $[1,2]$. In addition, long term medical care and loss of income create a considerable social and economical burden, which remains an unsolved public health problem [3]. Regrettably, to date there exists no clinically established therapy targeting the mechanisms involved in TBI and treatment remains limited to symptomatic approaches.

Dexmedetomidine is a highly selective $\alpha_{2}$-adrenoreceptor agonist with sedative, analgesic and sympatholytic properties. It was developed and investigated during the 1980s and 1990s and was initially used to achieve sedation and analgesia in veterinary medicine. In 1999 it was

\footnotetext{
* Correspondence: mcoburn@ukaachen.de

'Department of Anesthesiology, University Hospital of the RWTH Aachen,

Pauwelsstraße 30, 52074 Aachen, Germany Full list of author information is available at the end of the article
}

approved for use in humans by the US Food and Drug Administration for short-term sedation $(<24 \mathrm{~h})$ of patients in intensive care units and non-intubated patients prior to and during surgical procedures. Recently, dexmedetomidine was approved in Europe for sedation of adult intensive care unit patients requiring a sedation level not deeper than arousal in response to verbal stimulation.

Besides dexmedetomidine's well-proven sedative effects an increasing body of both in vitro and in vivo evidence indicates that dexmedetomidine also exerts a cell-protective effect on nervous tissue under ischemic conditions [4-7]. There is recent evidence suggesting that this effect is mediated not only by dexmedetomidine's $\alpha_{2}$-agonistic properties, but also by the binding to imidazoline $\mathrm{I}_{1}$-recepors [8]. The signal transduction cascade linked to these receptors comprises extracellular signal-regulated protein kinase 1 and 2 (ERK 1\&2) and is known to be an important regulator for cell survival and mediator of neuroprotective effects of various agents [9-11]. Nevertheless it has yet to be determined whether dexmedetomidine also
C Biomed Central 
promotes survival of nervous tissue that has suffered traumatic injury.

In this study we tested the hypothesis that dexmedetomidine has a protective effect on traumatically injured brain tissue as well. We used organotypic hippocampal brain-slice cultures subjected to a focal mechanical trauma as an in vitro model of TBI to analyse a potential neuroprotective effect of dexmedetomidine in TBI.

\section{Methods}

All experiments in the course of this study were approved by the animal protection representative at the Institute of Animal Research at RWTH Aachen University Hospital, according to the German animal protection law $\$ 4$, Section 3. Unless otherwise stated all chemicals were obtained from PAA Laboratories GmbH (Pasching, Austria).

\section{Hippocampal slice culture}

The organotypic hippocampal slice cultures were prepared from brains of 5-7 day old C57/BL6 mice pups using a well-established technique [12-14]. After decapitation of the pups the brains were quickly extracted from the sculls and placed into ice cold preparation medium comprising Gey's balanced salt solution (Sigma Aldrich, Steinheim, Germany), 5 mg/ml D-(+)-glucose (Roth, Karlsruhe, Germany) and $0.1 \%$ antibiotic/antimycotic solution (containing penicillin $\mathrm{G} 10,000 \mathrm{U} / \mathrm{ml}$, streptomycin sulphate $10 \mathrm{mg} / \mathrm{ml}$ and amphotericin B $25 \mu \mathrm{g} / \mathrm{ml}$ ).

The hippocampi were then dissected from the brains under stereomicroscopic vision, cut into $400 \mu \mathrm{m}$ thick slices and dispersed onto the semi-permeable membrane of MilliCell tissue culture inserts (MilliCell-CM, Millipore Corporation, Billerica, MA, USA). These inserts were placed inside $35 \mathrm{~mm}$ tissue culture plates (Sarstedt, Newton, MA, USA) and $1 \mathrm{ml}$ of growth medium (50\% Eagle minimal essential medium with Earle's salts, 25\% Hank's balanced salt solution (Sigma-Aldrich, Steinheim, Germany), 25\% heat inactivated horse serum, $2 \mathrm{mM} \mathrm{L}$-glutamine, $5 \mathrm{mg} / \mathrm{ml} \mathrm{D}$-glucose, $1 \%$ antibiotic/antimycotic solution and $50 \mathrm{mM}$ HEPES (hydroxyethyl-piperazineethanesulfonic acid) buffer solution (Sigma-Aldrich, Munich, Germany), titrated to $\mathrm{pH}$ 7.2) was instilled under the membranes to ensure optimal nutrition of the slices. The slices were then incubated for 14 days at $37^{\circ} \mathrm{C}$ and a moist atmosphere of $95 \%$ air and $5 \% \mathrm{CO}_{2}$. The growth medium was replaced $24 \mathrm{~h}$ after preparation and on every third day thereafter.

\section{Traumatic brain injury protocol}

On the day of the experiment growth medium was exchanged for experimental medium containing $4.5 \mu \mathrm{M}$ propidium Iodide. Experimental medium differed from growth medium only in the substitution of horse serum for more Eagle minimal essential medium assuring a standardized experimental environment for all slices. The slices were then incubated for $30 \mathrm{~min}$ at $37^{\circ} \mathrm{C}$ with a moist atmosphere of $95 \%$ air and $5 \% \mathrm{CO}_{2}$ before capturing baseline fluorescence images. Slices were then traumatized using a specially designed apparatus that ensured an equal and reproducible trauma during the course of the experiments $[15,16]$. For this purpose a metal stylus was lifted 7 $\mathrm{mm}$ above the slice with the aid of an electromagnet. When power to the magnet was switched off the stylus fell onto the slices producing a mechanical trauma of $5.26 \mu \mathrm{J}$ onto the hippocampus's CA1 region. The stylus was retrieved immediately after the impact and experimental medium was exchanged for new experimental medium in those plates belonging to the positive control group. In the experimental groups dexmedetomidine (Precedex ${ }^{\circledR} 100$ $\mu \mathrm{g} / \mathrm{ml}$ Dexmedetomidine, Orion Pharma, Espoo, Finland) was added to this experimental medium in various concentrations $(100 \mu \mathrm{M}, 10 \mu \mathrm{M}, 5 \mu \mathrm{M}, 1 \mu \mathrm{M}, 0.1 \mu \mathrm{M}, 0.01$ $\mu \mathrm{M})$. Concentrations were prepared based upon a molar weight of $236.7 \mathrm{~g} / \mathrm{mol}$ for dexmedetomidine- $\mathrm{HCl}$ as a basis. For the negative control group (non-traumatized slices), data from a previous, methodically identical study was used [12].

To further investigate the protective properties of dexmedetomidine, a series of other experiments was performed using a $1 \mu \mathrm{M}$ concentration which in the dose-finding study had been proven to be the most effective dose. To assess possible neuroprotective effects in a delayed application, experiments were repeated for $1 \mu \mathrm{M}$ dexmedetomidine, exchanging experimental medium at 2 and $3 \mathrm{~h}$ after the trauma, respectively. In order to verify the hypothesis that dexmedetomidine's neuroprotective properties involve activation of ERK the mitogen-activated/extracellular signal-regulated protein kinase kinase 1 (MEK1) -inhibitor PD98059 (Cell Signaling Technology, Danvers, MA, USA) was added in a $5 \mu \mathrm{M}$ concentration to the experimental medium containing $1 \mu \mathrm{M}$ dexmedetomidine.

All slices were incubated for $72 \mathrm{~h}$ at $37^{\circ} \mathrm{C}$ and a moist atmosphere of $95 \%$ air and $5 \% \mathrm{CO}_{2}$ after trauma.

In order to compare the effects of dexmedetomidine on cell survival with those of hypothermia, a positive control group of slices was incubated at $32^{\circ}$ and a moist atmosphere of $95 \%$ air and $5 \% \mathrm{CO}_{2}$ for $72 \mathrm{~h}$ after trauma. An experimental group exposed to $1 \mu \mathrm{M}$ of Dexmedetomidine was also incubated at $32^{\circ} \mathrm{C}$ for $72 \mathrm{~h}$ after trauma to study a possible synergistic effect of dexmedetomidine and hypothermia.

\section{Quantification of cell death and statistical analysis}

After the incubation period final fluorescence images were taken.

To quantify cell death propidium iodide was present in the media throughout all experiments. Propidium 
iodide is a widely used dyeing agent able to bind to nuclear DNA in a non-specific manner. Upon binding it becomes highly fluorescent with a peak emission in the spectrum of visible red light. Its properties render it incapable of penetrating intact phospholipids bilayers such as the cell membrane and the nuclear membrane. However, if the integrity of the cellular membranes is disrupted as in apoptotic or necrotic processes, propidium iodide can diffuse into the nucleus and stain the DNA.

Baseline and final fluorescence images were taken using an upright fluorescence microscope (Zeiss Axioplan, Carl Zeiss MicroImaging GmbH, Jena, Germany) equipped with a rhodamine filter and a low-power $\mathrm{x} 4$ objective lens (Zeiss Achroplan 4x/0.10, Carl Zeiss MicroImaging GmbH, Jena, Germany) and captured with a digital camera (SPOT Pursuit 4 MP Slider, Diagnostic Instruments Inc, Sterling Heights, MI, USA) and applicable software.

To eliminate any influence of the mercury lamp's fluctuating intensity, the exposure time was adapted before each imaging session using a standard fluorescence slice (Fluor-Ref, Omega Optical, Brattleboro, VT, USA) as reference.
ImageJ software was used to analyze the images. The fluorescence intensity of each pixel of the red channel was broken down to an eight bit value (between 0 and 255). Areas with high fluorescence values were equivalent to high propidium iodide uptake, thus strong cell damage. A histogram was then created showing the distribution of intensities within the image. Results of previously conducted studies using the same method $[12,13,17]$ point out that values below a threshold of 100 represent background fluorescence. Therefore, only values beyond this threshold were summarized and this integral served as a quantitative measure for cell death (Figure 1).

\section{Statistical analysis}

Mean \pm SEM (standard error of the mean) was calculated for each group using SPSS $19\left(\right.$ IBM $^{\circledR}$ SPSS $^{\circledR}$ Statistics, IBM Corporation, Somers, NY, USA). The fluorescence value of the positive control group was set to unity $(1 \pm 0.037)$ and served as a reference to put the observed effect of dexmedetomidine on cell survival into perspective. Values of all other groups were normalized to the positive-control group. Statistical relevance of the results was assessed performing an analysis of variance

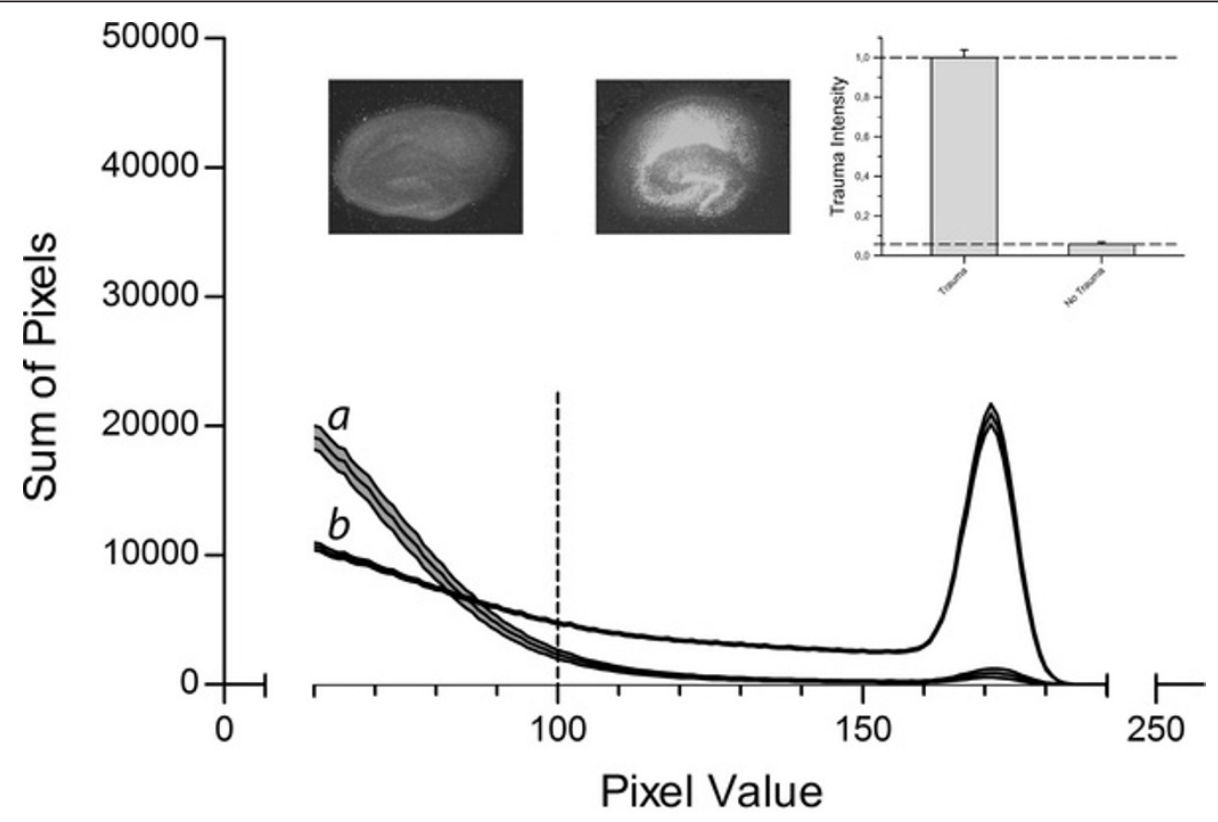

Figure 1 Control Data. Slices were cultivated for 14 days before being subjected to TBI introduced by a stylus dropped onto the CA1 region. Slices were then incubated in an atmosphere of $95 \%$ air and $5 \% \mathrm{CO}_{2}$. Negative control group slices were treated the same way, except for the trauma. After $72 \mathrm{~h}$ trauma intensity was assessed by fluorescence imaging and pixel based analysis. The fluorescence intensity corresponded to the trauma intensity of each pixel and was broken down to a value between 0 and 255. The histogram shows the distribution of pixels for the different fluorescence intensities for the negative control group $(n=35$, labelled $a)$ and the slices of the positive control group $(n=185$, labelled b). The middle line represents the mean for each fluorescence value; the upper and lower lines represent the upper and lower boundaries of the SEM. The interrupted vertical line is the applied threshold at a grey scale value of 100. The sum of all pixels beyond this threshold was calculated for each group and served as a measure for trauma intensity. Above the graph, example images for the negative control group (left) and positive control group (right) are shown. The insert in the upper right corner represents the trauma levels of both control groups normalized to the positive control group. 
(ANOVA). $P \leq 0.05$ was considered statistically significant.

\section{Results}

As Figure 2 points out the concentrations $10 \mu \mathrm{M}, 5 \mu \mathrm{M}$, $1 \mu \mathrm{M}$ and $0.1 \mu \mathrm{M}$ showed a significant reduction in trauma intensity with a maximum effect at $1 \mu \mathrm{M}$, where the fluorescent value was $42.9 \%$ of the positive control group's value. No significant change in trauma intensity was observed at $100 \mu \mathrm{M}(1.094 \pm 0.065$ vs. $1 \pm 0.037$, $P=0.213)$ and $0.01 \mu \mathrm{M}(0.949 \pm 0.061$ vs. $1 \pm 0.037$, $P=0.485)$.

While hypothermia proved to be neuroprotective when comparing its effects to the positive control group $(0.659$ \pm 0.074 vs. $1 \pm 0.037, P<0.001$ ), hypothermia was associated with significantly less neuroprotection than dexmedetomidine at a concentration of $1 \mu \mathrm{M}(0.659 \pm 0.074$ vs. $0.429 \pm 0.046, P=0.008$ ). The simultaneous application of hypothermia and $1 \mu \mathrm{M}$ dexmedetomidine was neither significantly superior nor inferior to the application of $1 \mu \mathrm{M}$ dexmedetomidine alone $(0.578 \pm 0.064$ vs. $0.429 \pm 0.046, P=0.073$ ) (Figure 3).

Also, the delayed application of dexmedetomidine $(2 \mathrm{~h}$ and $3 \mathrm{~h}$ after trauma, respectively) showed a protective effect. Application of dexmedetomidine $2 \mathrm{~h}$ after trauma

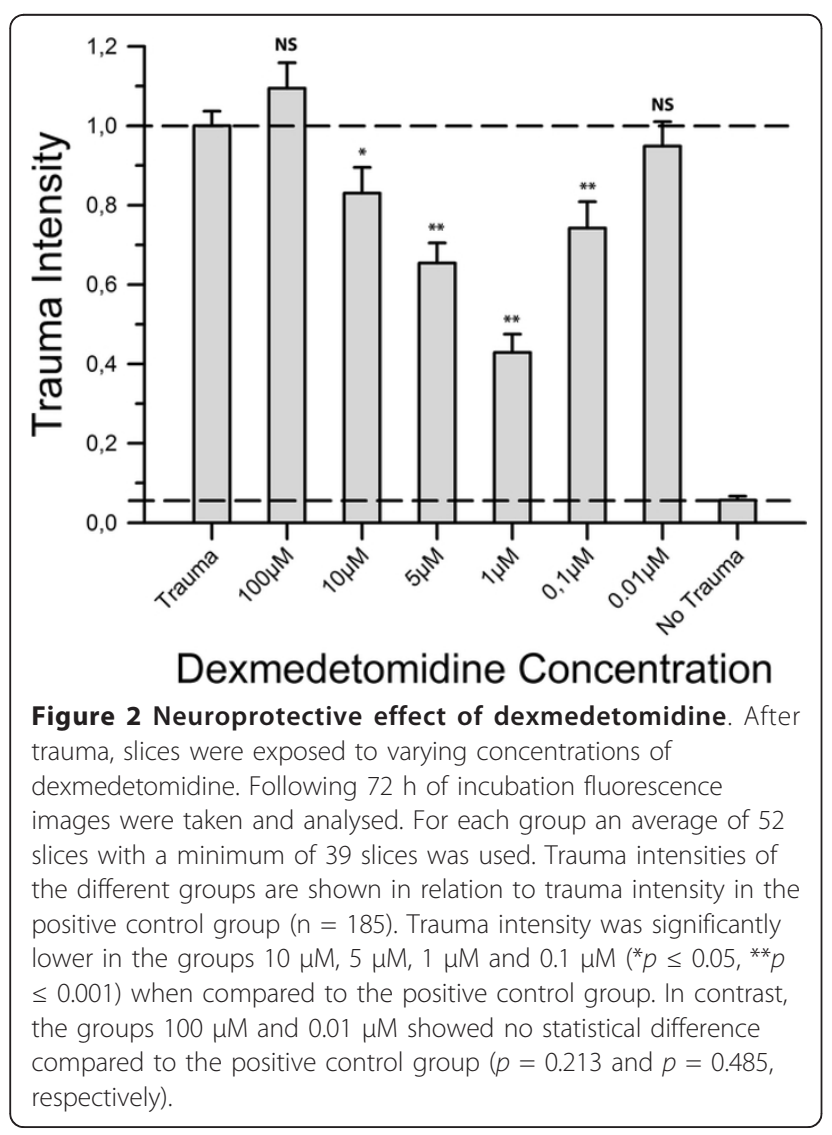

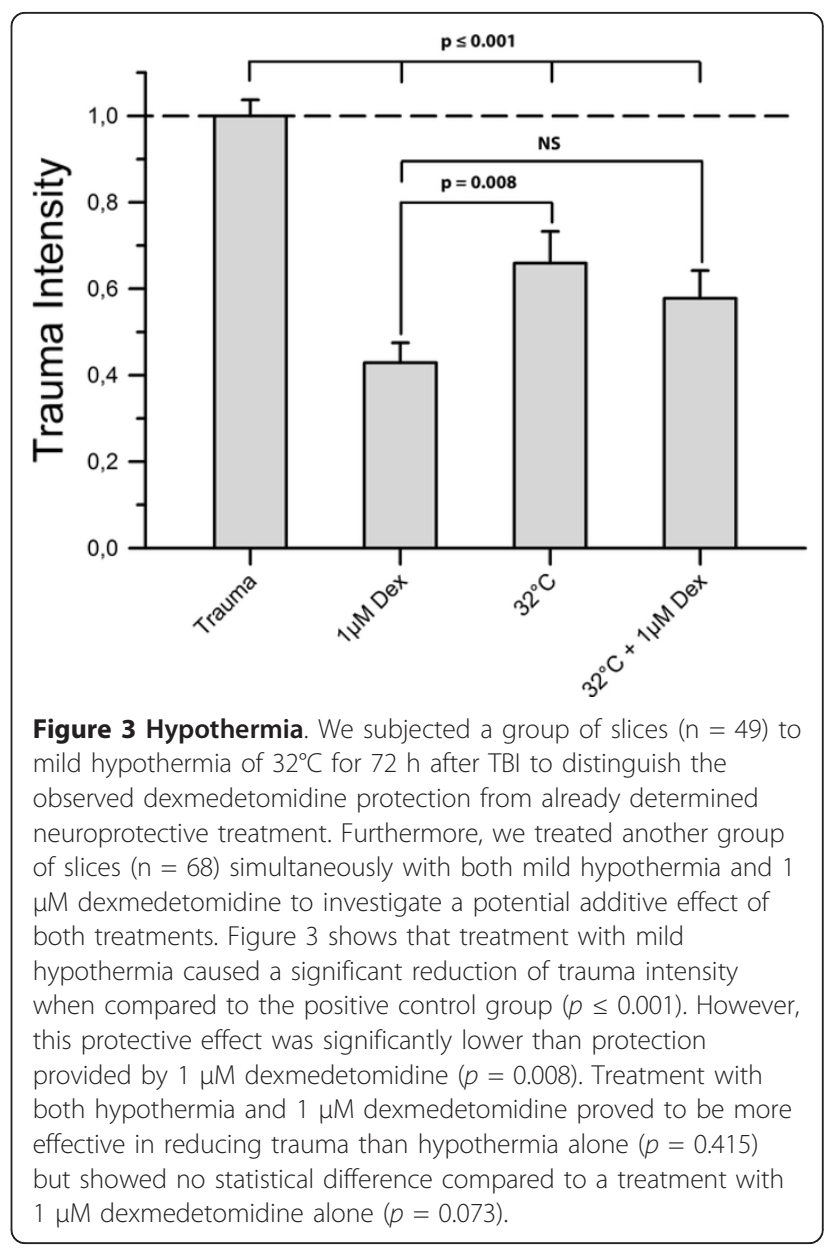

proved even more efficient than immediate application $(0.27 \pm 0.028$ vs. $0.429 \pm 0.046, P=0.030)$ while a delay of three hours $(0.363 \pm 0.04$ vs. $0.429 \pm 0.046, P=$ 0.335 ) resulted in similar effects as the instantaneous application (Figure 4).

The simultaneous application of $1 \mu \mathrm{M}$ dexmedetomidine and PD98059 resulted in a statistically significant reduction of cellular protection $(0.578 \pm 0.051$ vs. $0.429 \pm$ $0.046, P=0.033$ ) (Figure 5).

\section{Discussion}

The aim of this study was to investigate the effects of the $\alpha_{2}$-adrenoreceptor agonist dexmedetomidine on cell survival in an established in vitro model for TBI. Using organotypic hippocampal slice cultures subjected to a focal mechanical trauma we found that dexmedetomidine had a dose-dependent protective effect on hippocampal cells. The dose-effect curve was U-shaped, with a concentration of $1 \mu \mathrm{M}$ showing the strongest effect. Moreover, we observed neuroprotection by dexmedetomidine even when applied with a delay after the onset of the traumatic injury. Apparently, the neuroprotective effects of 

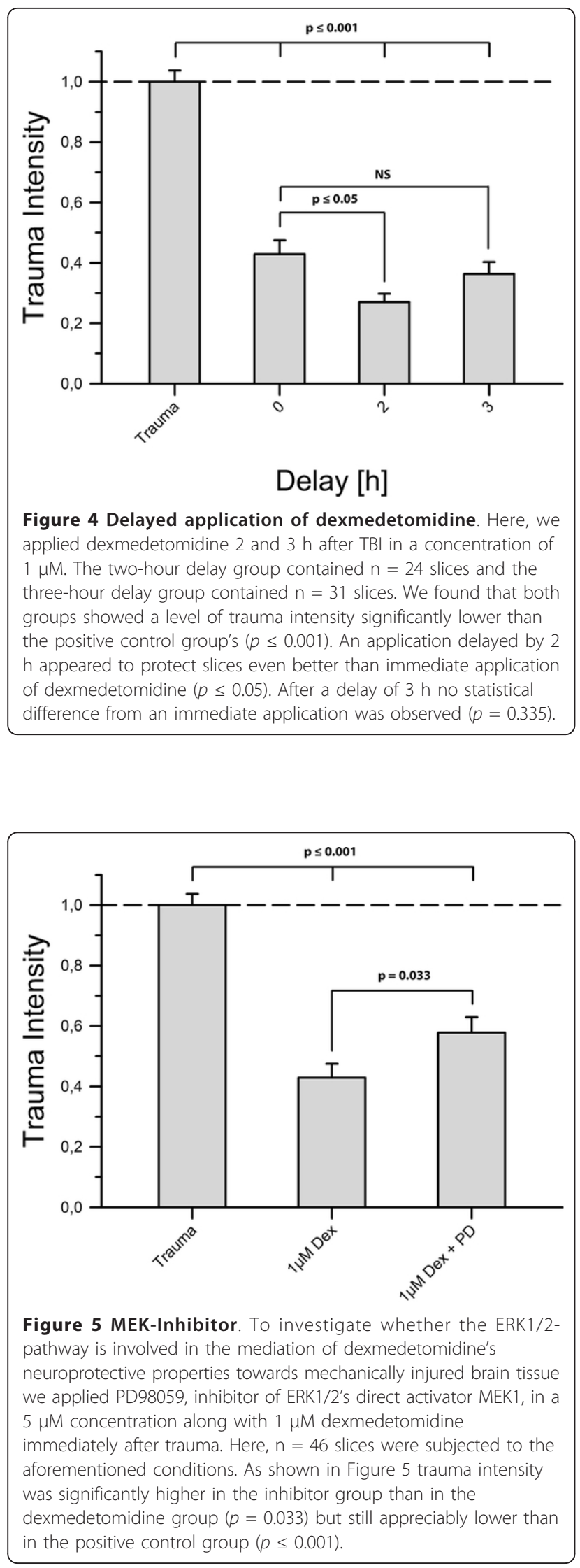

dexmedetomidine are mediated - at least in part - by ERK as they were abolished by the co-administration of PD98059. Interestingly, the co-application of hypothermia and dexmedetomidine did not exert synergistic effects.

We treated experimental groups with varying concentrations of dexmedetomidine after trauma. Results show that a concentration of $1 \mu \mathrm{M}$ dexmedetomidine was most effective at reducing trauma intensity. This finding is consistent with data from a recent study using hippocampal slice culture subjected to oxygen and glucose deprivation [5]. Further dilution as well as a higher concentration of dexmedetomidine provided lower protection until finally, at $100 \mu \mathrm{M}$ and $0.01 \mu \mathrm{M}$, no difference compared to the positive control group was observed. Comparable observations have been made in other studies investigating dexmedetomidine's protective properties $[5,18]$.

Dexmedetomidine's neuroprotective properties have largely been attributed to its agonist actions at $\alpha_{2}$-adrenoreceptors $[19,20]$. Although recent investigations point out that its interaction with imidazoline I1 receptors and the activation of ERK might also play a role $[5,8,21]$ we did not explicitly analyze imidazoline $I_{1}$ receptors. However, the results of our experiments indicate that activation of ERK is an important factor in the protection of traumatized nervous tissue by dexmedetomidine. ERK is a key enzyme in cell metabolism activated by many different types of tissue injury and has been attributed a "survival"function. The inhibition of a survival signals itself can cause cells to undergo apoptosis; therefore application of PD98059, inhibitor of ERK's direct activator MEK1, might as well have had a negative effect on cellular survival that superposed with the positive effect of dexmedetomidine without specifically counteracting dexmedetomidine. To rule out this possibility western blots could have been performed. Comparison of levels of activated ERK for the positive control group, the $1 \mu \mathrm{M}$ dexmedetomidine group and the $1 \mu \mathrm{M}$ dexmedetomidine + PD98059 group could have backed up our hypothesis. This has recently been performed in a similarly designed study investigating dexmedetomidine's neuroprotective properties using organotypic hippocampal slice cultures subjected to oxygen and glucose deprivation. Results of this study showed that dexmedetomidine's and PD98059's effects do not simply superpose but that both act as two opponents on the same cascade [5]. We therefore decided not to perform these tests and interpreted our finding as a sufficient hint towards an involvement of ERK in the mediation of the brain protective properties of dexmedetomidine in TBI.

Many experimental studies have revealed neuroprotective properties of hypothermia $[13,16,22,23]$ and the advantages of hypothermia in certain clinical circumstances have been demonstrated [24]. To put our findings into context we subjected a group of slices to mild 
hypothermia of $32^{\circ} \mathrm{C}$ from trauma until final imaging. As expected, hypothermia alone did prove to be strongly protective, but $1 \mu \mathrm{M}$ dexmedetomidine was even more effective at reducing trauma intensity. Interestingly, as described before in in vivo models of incomplete cerebral ischemia, a combination of hypothermia and $1 \mu \mathrm{M}$ dexmedetomidine did not result in synergistic effects [7].

In a clinical setting, unpredictability is in the nature of TBI and application of any specific therapy will inevitably be delayed. Therefore we investigated the effect of dexmedetomidine when applied 2 or $3 \mathrm{~h}$ after trauma. In our model dexmedetomidine proved to be even more efficient when application was delayed by $2 \mathrm{~h}$. However, by delaying application for $3 \mathrm{~h}$ no appreciable difference could be observed any more compared to immediate application.

We acknowledge that our results should be interpreted within the context of several limitations.

Propidium iodide labels the DNA of any disrupted cell and we were therefore not able to distinguish between necrosis and apoptosis. However, we aimed to quantify the total amount of cell death and it is agreed that propidium iodide uptake correlates well with the number of damaged cells $[15,25]$.

We opted for organotypic slice cultures because this model allows easy access to in vitro manipulation of nervous tissue and yet mimics closely the in vivo state of the tissue with respect to morphological and functional characteristics [26,27]. Concerning the extrapolation to an in vivo situation, results from organotypic slice cultures have been demonstrated to be a satisfying compromise between dissociated cell culture and in vivo models using whole animals $[28,29]$. We admit, however, that extrapolation of our results to the in vivo situation is complex. In a living organism, the development and the outcome from TBI is significantly affected by a plethora of different variables that can only partly, if at all, be taken into consideration in an in vitro model (e.g. cerebral perfusion pressure, edema of surrounding tissue, focal or global cerebral ischemia). Such conditions may be affected by dexmedetomidine independently from its direct effects on neuronal survival. Despite these disadvantages we believe that our model is appropriate as it focuses on the mechanical component of the injury and allows the analysis of the intrinsic neuroprotective properties of a drug independently from its possible interactions with confounding variables that can only poorly be controlled in an in vivo setting.

\section{Conclusion}

We have found that dexmedetomidine has a protective effect on hippocampal slice cultures subjected to a focal mechanical trauma. The maximum effect was observed at $1 \mu \mathrm{M}$, with the observed trauma reduction being significantly more pronounced than observed in slices treated with hypothermia. Trauma intensity did not further decline when applying dexmedetomidine and hypothermia simultaneously. Also, dexmedetomidine's effect was partially reversed by simultaneous application of MEK1 Inhibitor PD98059 indicating the involvement of the ERKsignalling cascade.

\section{Key messages}

- Dexmedetomidine exerts a neuroprotective effect when administered after TBI in this model of organotypic hippocampal slice cultures

- The protective effect of dexmedetomidine is significantly stronger than the effect of mild hypothermia at $32^{\circ} \mathrm{C}$

- Dexmedetomidine has a protective effect even if application after TBI is delayed by 2 and $3 \mathrm{~h}$, respectively

- Activation of ERK might be involved in mediating Dexmedetomidine's protective effect

\section{Abbreviations}

TBI: Traumatic brain injury; ERK: Extracellular signal-regulated protein kinase; HEPES: Hydroxyethyl-piperazine-ethanesulfonic acid; MEK: Mitogen-activated/ extracellular signal-regulated protein kinase kinase; SEM: Standard error of the mean; ANOVA: Analysis of variance.

\section{Acknowledgements}

We would like to thank Rosemarie Blaumeiser-Debarry and Jenny Hoffmann for their help with data acquisition as well as the Departments of Neuropathology, Pathology and Animal Research teams at the University Hospital Aachen for expert laboratory advice and assistance. Finally we would like to thank Monroe Coburn for editorial suggestions.

\section{Author details}

'Department of Anesthesiology, University Hospital of the RWTH Aachen, Pauwelsstraße 30, 52074 Aachen, Germany. ' Institute of Neuropathology, University Hospital of the RWTH Aachen, Pauwelsstraße 30, 52074 Aachen, Germany. ${ }^{3}$ Department of Anesthesiology, University Hospital of the KU Leuven, Herestraat 49, 3000 Leuven, Belgium.

\section{Authors' contributions}

MS conducted the experimental laboratory work, performed the statistical analysis and drafted the manuscript. AVF, GE, RR and SR participated in the study design and coordination and helped to draft the manuscript. PDL and JW helped to perform the study and assisted in drafting the manuscript. MC originated the study, participated in its study design and coordination and helped to draft the manuscript. All authors read and approved the final text.

\section{Competing interests}

This study was supported by Orion Corporation, Espoo, Finland. $\mathrm{RR}$ received consultant fees from Orion Corporation, a company producing and marketing pharmaceuticals such as dexmedetomidine. All other authors declare that they have no competing interests.

Received: 25 October 2011 Accepted: 11 April 2012 Published: 11 April 2012

\section{References}

1. Corrigan JD, Selassie AW, Orman JA: The epidemiology of traumatic brain injury. J Head Trauma Rehabil 2010, 25:72-80.

2. Selassie AW, Zaloshnja E, Langlois JA, Miller T, Jones P, Steiner C: Incidence of long-term disability following traumatic brain injury hospitalization, United States, 2003. J Head Trauma Rehabil 2008, 23:123-131.

3. Thurman DJ, Alverson C, Dunn KA, Guerrero J, Sniezek JE: Traumatic brain injury in the United States: a public health perspective. I Head Trauma Rehabil 1999, 14:602-615. 
4. Hoffman WE, Kochs E, Werner C, Thomas C, Albrecht RF: Dexmedetomidine improves neurologic outcome from incomplete ischemia in the rat. Reversal by the alpha 2-adrenergic antagonist atipamezole. Anesthesiology 1991, 75:328-332.

5. Dahmani S, Rouelle D, Gressens P, Mantz J: Characterization of the postconditioning effect of dexmedetomidine in mouse organotypic hippocampal slice cultures exposed to oxygen and glucose deprivation. Anesthesiology 2010, 112:373-383.

6. Engelhard K, Werner C, Eberspacher E, Bachl M, Blobner M, Hildt E, Hutzler $P$, Kochs $E$ : The effect of the alpha 2-agonist dexmedetomidine and the $\mathrm{N}$-methyl-D-aspartate antagonist $\mathrm{S}(+)$-ketamine on the expression of apoptosis-regulating proteins after incomplete cerebral ischemia and reperfusion in rats. Anesth Analg 2003, 96:524-531.

7. Sato K, Kimura T, Nishikawa T, Tobe Y, Masaki Y: Neuroprotective effects of a combination of dexmedetomidine and hypothermia after incomplete cerebral ischemia in rats. Acta Anaesthesiol Scand 2010, 54:377-382.

8. Dahmani S, Paris A, Jannier V, Hein L, Rouelle D, Scholz J, Gressens P, Mantz J: Dexmedetomidine increases hippocampal phosphorylated extracellular signal-regulated protein kinase 1 and 2 content by an alpha 2-adrenoceptor-independent mechanism: evidence for the involvement of imidazoline I1 receptors. Anesthesiology 2008, 108:457-466.

9. Toborek M, Son KW, Pudelko A, King-Pospisil K, Wylegala E, Malecki A: ERK $1 / 2$ signaling pathway is involved in nicotine-mediated neuroprotection in spinal cord neurons. J Cell Biochem 2007, 100:279-292.

10. Shen J, Wu Y, Xu JY, Zhang J, Sinclair SH, Yanoff M, Xu G, Li W, Xu GT: ERKand Akt-dependent neuroprotection by erythropoietin (EPO) against glyoxal-AGEs via modulation of $\mathrm{Bcl}-\mathrm{xL}$, Bax, and BAD. Invest Ophthalmol Vis Sci 2010, 51:35-46.

11. Troadec JD, Marien M, Mourlevat S, Debeir T, Ruberg M, Colpaert F, Michel PP: Activation of the mitogen-activated protein kinase (ERK(1/2)) signaling pathway by cyclic AMP potentiates the neuroprotective effect of the neurotransmitter noradrenaline on dopaminergic neurons. $\mathrm{Mol}$ Pharmacol 2002, 62:1043-1052.

12. Loetscher PD, Rossaint J, Rossaint R, Weis J, Fries M, Fahlenkamp A Ryang YM, Grottke O, Coburn M: Argon: neuroprotection in in vitro models of cerebral ischemia and traumatic brain injury. Crit Care 2009, 13:R206.

13. Rossaint J, Rossaint R, Weis J, Fries M, Rex S, Coburn M: Propofol: neuroprotection in an in vitro model of traumatic brain injury. Crit Care 2009, 13:R61.

14. Stoppini $L$, Buchs PA, Muller D: A simple method for organotypic cultures of nervous tissue. J Neurosci Methods 1991, 37:173-182.

15. Adamchik Y, Frantseva MV, Weisspapir M, Carlen PL, Perez Velazquez JL: Methods to induce primary and secondary traumatic damage in organotypic hippocampal slice cultures. Brain Res Brain Res Protoc 2000, 5:153-158

16. Coburn M, Maze M, Franks NP: The neuroprotective effects of xenon and helium in an in vitro model of traumatic brain injury. Crit Care Med 2008, 36:588-595.

17. Roehl $A B$, Hein M, Loetscher PD, Rossaint J, Weis J, Rossaint R, Coburn M: Neuroprotective properties of levosimendan in an in vitro model of traumatic brain injury. BMC Neurol 2010, 10:97.

18. Laudenbach V, Mantz J, Lagercrantz H, Desmonts JM, Evrard P, Gressens P: Effects of alpha(2)-adrenoceptor agonists on perinatal excitotoxic brain injury: comparison of clonidine and dexmedetomidine. Anesthesiology 2002, 96:134-141.

19. Ma D, Hossain M, Rajakumaraswamy N, Arshad M, Sanders RD, Franks NP, Maze M: Dexmedetomidine produces its neuroprotective effect via the alpha 2A-adrenoceptor subtype. Eur J Pharmacol 2004, 502:87-97.

20. Paris A, Mantz J, Tonner PH, Hein L, Brede M, Gressens P: The effects of dexmedetomidine on perinatal excitotoxic brain injury are mediated by the alpha2A-adrenoceptor subtype. Anesth Analg 2006, 102:456-461.

21. Sanders RD, Sun P, Patel S, Li M, Maze M, Ma D: Dexmedetomidine provides cortical neuroprotection: impact on anaesthetic-induced neuroapoptosis in the rat developing brain. Acta Anaesthesiol Scand 2010, 54:710-716.

22. Hua $Y$, Hisano K, Morimoto Y: Effect of mild and moderate hypothermia on hypoxic injury in nearly pure neuronal culture. J Anesth 2010, 24:726-732.

23. Mueller-Burke D, Koehler RC, Martin L: Rapid NMDA receptor phosphorylation and oxidative stress precede striatal neurodegeneration after hypoxic ischemia in newborn piglets and are attenuated with hypothermia. Int J Dev Neurosci 2008, 26:67-76.

24. The hypothermia after cardiac arrest study group: Mild therapeutic hypothermia to improve the neurologic outcome after cardiac arrest. $N$ Engl J Med 2002, 346:549-556.

25. Tasker RC, Coyle JT, Vornov JJ: The regional vulnerability to hypoglycemia-induced neurotoxicity in organotypic hippocampal culture: protection by early tetrodotoxin or delayed MK-801. J Neurosci 1992, 12:4298-4308.

26. Finley M, Fairman D, Liu D, Li P, Wood A, Cho S: Functional validation of adult hippocampal organotypic cultures as an in vitro model of brain injury. Brain Res 2004, 1001:125-132

27. Muller D, Buchs PA, Stoppini L: Time course of synaptic development in hippocampal organotypic cultures. Brain Res Dev Brain Res 1993, 71:93-100

28. Morrison B, Saatman KE, Meaney DF, Mclntosh TK: In vitro central nervous system models of mechanically induced trauma: a review. J Neurotrauma 1998, 15:911-928.

29. Noraberg J, Poulsen FR, Blaabjerg M, Kristensen BW, Bonde C, Montero M, Meyer M, Gramsbergen JB, Zimmer J: Organotypic hippocampal slice cultures for studies of brain damage, neuroprotection and neurorepair. Curr Drug Targets CNS Neurol Disord 2005, 4:435-452.

\section{Pre-publication history}

The pre-publication history for this paper can be accessed here: http://www.biomedcentral.com/1471-2377/12/20/prepub

doi:10.1186/1471-2377-12-20

Cite this article as: Schoeler et al:: Dexmedetomidine is neuroprotective in an in vitro model for traumatic brain injury. BMC Neurology 2012 12:20.

\section{Submit your next manuscript to BioMed Central and take full advantage of:}

- Convenient online submission

- Thorough peer review

- No space constraints or color figure charges

- Immediate publication on acceptance

- Inclusion in PubMed, CAS, Scopus and Google Scholar

- Research which is freely available for redistribution 